\title{
Quaderni
}

QUADERNI Communication, technologies, pouvoir

76 | Automne 2011

Les promesses de la biodiversité

\section{La fabrique de l'information : le cas des grandes expéditions naturalistes contemporaines}

\section{Elsa Faugère et Olivier Pascal}

\section{(2) OpenEdition}

\section{Édition électronique}

URL : http://journals.openedition.org/quaderni/101

DOI : 10.4000/quaderni.101

ISSN : 2105-2956

\section{Éditeur}

Les éditions de la Maison des sciences de l'Homme

\section{Édition imprimée}

Date de publication : 1 septembre 2011

Pagination : $39-51$

\section{Référence électronique}

Elsa Faugère et Olivier Pascal, « La fabrique de l'information : le cas des grandes expéditions naturalistes contemporaines », Quaderni [En ligne], 76 | Automne 2011, mis en ligne le 01 septembre 2014, consulté le 01 mai 2019. URL : http://journals.openedition.org/quaderni/101 ; DOI : 10.4000/ quaderni.101 


\section{$D$ ossier}

\section{la fabrique de l'information : \\ le cas des \\ grandes \\ expéditions naturalistes \\ contemporaines}

Depuis les années 2000, des expéditions naturalistes qualifiées par les médias « de plus grandes de tous les temps » sont organisées par des organismes français spécialistes des questions d'exploration scientifique et de conservation de la biodiversité ou plus généralement d'environnement.

Avant les années 2000 et aujourd'hui encore, les naturalistes professionnels ou amateurs partent régulièrement en mission pour effectuer des observations sur les espèces et des collectes de spécimens. Mais ces «petites » missions n'ont aucun écho médiatique. Parmi les milliers de nouvelles espèces ainsi découvertes et décrites chaque année dans les revues scientifiques, quasiment aucune - sauf parfois une nouvelle espèce d'un animal emblématique comme un mammifère ou un oiseau - ne parvient à figurer dans les médias à titre « individuel ». L'exploration de la biodiversité et son corollaire, la découverte

Elsa de nouvelles espèces, font rarement la Une des Faugère journaux français.

Anthropologue, INRA, Ecodéveloppement

Olivier Pascal

Botaniste, Pro-Natura International
Plus globalement, comme le souligne Françoise Tristani-Potteaux (1997: 1) : "une découverte scientifique a beaucoup moins de chances de faire les gros titres des journaux qu'une courbe de chômage, une grève des cheminots ou une allocution présidentielle ».

Pourtant en 2006 eut lieu au Vanuatu une grande expédition naturaliste appelée Santo 2006 organisée par le Muséum National d'Histoire Naturelle de Paris, Pro-Natura International et l'Institut de Recherche pour le Développement (IRD) - qui reçut une couverture médiatique sans précédent pour ce genre d'opération. Deux ans 
plus tôt, en 2004, une grande expédition marine, organisée aux Philippines par le Muséum n'avait bénéficié d'aucune retombée dans les grands médias français. En 2009/2010, une nouvelle expédition naturaliste, dans la lignée de l'expédition Santo 2006, l'expédition Mozambique/ Madagascar ${ }^{1}$ bénéficie elle aussi d'une importante couverture médiatique.

Que s'est-il passé, dans un si court laps de temps, pour que des opérations scientifiques comparables sur de nombreux aspects bénéficient d'une couverture médiatique aussi différente?

Nous ferons l'hypothèse que c'est l'existence d'une stratégie et d'un plan de communication professionnels, émanant des organisateurs de ces grandes expéditions, qui permettent le mieux de comprendre cette soudaine médiatisation. L'alliance du Muséum National d'Histoire Naturelle de Paris avec un partenaire privé (Pro-Natura International) rodé à la communication a été conclue dans la seconde moitié des années 2000, au moment où le Muséum, comme la plupart des grands instituts de recherche français, modernisait et professionnalisait sa Direction de la Diffusion et de la Communication devenue, en 2005, la Direction de la Diffusion, de la Communication, de l'Accueil et des Partenariats (DICAP).

Cette professionnalisation des stratégies et des services de communication des institutions en charge des grandes expéditions naturalistes s'effectue de manière concomitante au développement, en France, d'un mécénat privé. Hors coût salarial, ces expéditions naturalistes sont financées par des mécènes qui en attendent un retour d'images ${ }^{2}$. Mécénat, communication et médiatisation sont étroitement imbriqués. Ce qui est présenté dans les médias et dans les dossiers de presse élaborés par les services de la communication du Muséum, comme "le renouveau des grandes expéditions naturalistes » bénéficie donc non seulement de ces nouveaux métiers de la communication, du soutien de grands mécènes privés mais aussi d'un nouvel intérêt des médias pour les questions liées à l'environnement.

Dans les années 2000, alors que les médias traversent une crise majeure, l'espace alloué à l'actualité environnementale augmente considérablement grâce à la création de rubriques régulières et de (sous-) services dédiés, comme le montre Jean-Baptiste Comby (2009 : 165). «L'environnement devient médiatique », le journalisme environnemental s'institutionnalise et, ce faisant, il perd le caractère engagé et militant qu'il possédait depuis la fin des années 1960 (Comby 2009 : 165).

Mais le traitement médiatique de ces grandes expéditions naturalistes ne se situe pas exclusivement dans le domaine environnemental. Il est à l'interface avec le journalisme scientifique. Or, dans les années 2000, le journalisme scientifique est, quant à lui, en crise (Tristani-Potteaux 2001 : 7). C'est donc dans un contexte médiatique difficile que les organisateurs des expéditions naturalistes tentent d'élaborer des plans médias efficaces, condition sine qua non pour pérenniser le soutien financier des mécènes et les expéditions elles-mêmes.

L'objectif de cet article est d'étudier, non pas le journalisme scientifique et environnemental pour lui-même, mais la chaîne de production de 
l'information depuis les sources elles-mêmes jusqu'à leur traitement médiatique. Ces sources sont a priori diverses et variées. Or l'analyse des revues de presse montre, au contraire, que les mêmes sources sont systématiquement reprises par la grande majorité des médias (audiovisuels et écrits). «La sociologie des sources d'information a déjà été présentée comme un sujet de première importance " remarque Philip Schlesinger (1992 : 77) dans un article de référence sur la sociologie du journalisme. Vingt ans plus tard, le regret exprimé par Philip Schlesinger sur le manque d'attention accordée par la sociologie des médias aux relations entre médias et sources d'informations semble toujours d'actualité.

Dans une première partie, nous nous intéresserons au contenu des « stratégies » de communication des organisateurs de grandes expéditions depuis la fin des années 1980. Nous verrons qu'il y a eu une nette évolution en trente ans. Dans une seconde partie, nous nous centrerons sur la chaîne qui permet de fabriquer l'information, depuis les sources elles-mêmes jusqu'aux journalistes. Puis nous conclurons par un questionnement sur l'émergence d'une opinion publique en matière environnementale en général et en matière d'érosion de la biodiversité en particulier.

Il s'agit là d'un article exploratoire qui s'appuie sur deux enquêtes de terrain menées par Elsa Faugère (de 2005 à 2007 sur l'expédition Santo, et depuis janvier 2010 sur l'expédition Mozambique/Madagascar). L'article s'appuie aussi sur les connaissances et l'expérience d'Olivier Pascal, l'un des responsables de l'équipe du Radeau des Cimes dans les années 1990, salarié de l'ONG Pro-Natura International co-organisateur des ex- péditions Santo et Mozambique/Madagascar et qui a contribué au montage de leur volet médiatique.

Nous avons notamment fait une analyse des revues de presse des expéditions Panglao, Santo et Mozambique/Madagascar. La revue de presse de Panglao 2004 a été constituée par Philippe Maestrati, proche collaborateur de Philippe Bouchet, co-organisateur des expéditions étudiées ici ; celle de l'expédition Santo a été faite par le service de communication du Muséum (la DICAP) et celle de l'expédition Mozambique/Madagascar a été faite par une agence privée de communication, The Desk ${ }^{3}$.

Nous avons également analysé les sites internet et les dossiers de presse des expéditions suivantes : Le Radeau des Cimes (Madagascar 2000-2001), Panglao 2004, Clipperton 2004 (organisée par Jean-Louis Etienne), Santo 2006, Mozambique/ Madagascar 2009-2010, La Boudeuse 2009-2011 (organisée par Patrice Franceschi).

\section{« Héros de l'environnement » versus « scien- tifiques-explorateurs ordinaires »}

\section{Une tentative de typologie des opérations}

La comparaison des dossiers de presse et des sites internet des expéditions listées ci-dessus n'est ni exhaustive, ni représentative, mais elle est significative : elle montre l'existence, depuis la fin des années 1980, d'au moins deux grands types d'expéditions naturalistes, dont le succès, médiatique et auprès des mécènes, a varié au cours du temps :

- les expéditions qui, incarnées par une figure 
individuelle dotée d'une certaine notoriété médiatique, conduisent à une extrême personnalisation, voire à une quasi-héroïsation de la figure de l'explorateur-aventurier sur le modèle des expéditions de Jean-Louis Étienne et de Patrice Franceschi. Même si ces expéditions se revendiquent comme scientifiques, elles s'inscrivent dans une logique événementielle et de communication avec la production et la vente de multiples produits dérivés (films, livres, etc.) qui s'appuient d'abord sur la notoriété de l'explorateur-aventurier, avant celle de l'aventure elle-même.

À ces figures quasi héroïques des explorateurs des temps modernes fait face un autre type d'explorateurs et d'expéditions qui met en avant, non pas des personnes, mais des objectifs scientifiques et des engins (volants ou navigants) :

- les expéditions qui, sur le modèle des expéditions du « Radeau des Cimes » et de « La Planète Revisitée » ne sont pas portées par un personnage emblématique et médiatique mais s'incarnent dans - ou en tout cas mettent en avant - des objectifs scientifiques (inventaires et conservation de la biodiversité, questionnements écologiques, recherches sur le vivant en général, etc.) et des moyens technologiques exceptionnels (engins volants, navires de recherche, code-barres du vivant, etc.). Tout en étant, elles aussi, majoritairement financées par des mécènes privés (fondations, fondations d'entreprises, sociétés), ces expéditions ne s'inscrivent pas dans une logique de communication (même si cette dernière devient une composante essentielle de l'opération).

Les raisons du choix de l'une ou l'autre de ces stratégies sont multiples. Le choix est souvent fait par défaut : on choisira de mettre en avant son ou ses atouts dans la présentation d'un projet : un engin, dans le cas d'aventuriers « anonymes », une personnalité, lorsqu'elle détient une notoriété suffisante, des « objectifs » si l'on ne possède ni l'un ni l'autre.

\section{Des années 1980 aux années 2010 : de la figure emblématique à l'explorateur ordinaire}

La réussite de l'une ou l'autre de ces deux grandes stratégies de communication a varié au cours de ces trente dernières années. Au risque de caricaturer, on peut grossièrement distinguer deux grandes périodes ${ }^{4}$. La première, que l'on situe dans les années 1980/1990 correspond à une appétence (des bailleurs de fonds, des médias et du public) pour des opérations où le « contenant» domine le « contenu ». Il était relativement facile de monter des projets sur les seuls ressorts de l'aventure ou de la technique sans trop se préoccuper du fond, ni d'avoir à véritablement justifier des activités menées. Les projets n'étaient pas sans contenu mais celui-ci n'était pas l'argument déclencheur d'un financement ni l'élément clé de la couverture médiatique. C'est partiellement encore le cas, mais c'est l'équivalent d'un effet de traîne et ceux qui de nos jours favorisent cette approche (au moins pour les domaines qui touchent à la « Nature ») ont de plus en plus de difficultés à faire vivre leurs projets.

La deuxième période, depuis le début des années 2000, privilégie et préfère les projets du deuxième type, dans lesquels le « pourquoi faire » est plus important que le « faire », où le potentiel narratif existe. Les projets porteurs de questionnements scientifiques ou géographiques, par exemple, ont 
le vent en poupe.

Longtemps en concurrence amicale avec JeanLouis Étienne pour les mêmes « guichets », les promoteurs du Radeau des Cimes ont souvent entendu, comme argument d'une réponse négative à une demande de soutien financier, que leur aventure, à la différence de celle portée par JeanLouis Étienne, manquait d'une « figure », d'un nom qui - postulat de l'époque - permettrait au public d'identifier et de « situer» l'aventure. On peut y voir « l'effet Cousteau » qui a durablement marqué les esprits et dont la plupart des aventuriers modernes se revendiquent ouvertement. Le vedettariat était de mise.

Le « Radeau des Cimes », comme l'expédition Clipperton organisée en 2005 par Jean-Louis Étienne, étaient des missions scientifiques avec cependant comme particularité commune un programme scientifique sous forme d'un catalogue de projets, généralement individuels, sans ligne directrice. Cette juxtaposition de petits projets de qualités inégales, mais globalement valables lorsqu' on les passe en revue un par un - était difficile, voire impossible, à résumer sans tomber dans les travers d'un catalogue à la Prévert. Et aucun n'était suffisamment attractif en soi pour être mis particulièrement en avant. En conséquence, plutôt que le contenu, l'accroche mettait en avant des moyens (engins volants notamment), des lieux, etc. pour retenir une attention souvent brève et minutée : des partenaires financiers, les « DirCom »-dans des rendez-vous souvent bâclés - ; des partenaires médias, à l'affût du « coup », tous élevés dans la culture du « pitch», terme ingrat désignant « l'argument», le ressort dramatique, et qui consiste à résumer un projet en quelques phrases. Ce schéma fonctionnait principalement dans les années 1980/1990 même si Clipperton date du début des années 2000.

En 2006, lors de 1'expédition Santo au Vanuatu, les aventuriers « ordinaires » ont reçu les faveurs du public et de la presse. Le film réalisé sur l'expédition et diffusé dans l'émission grand public Thalassa, présentait non pas une figure emblématique, monopolisant l'écran, mais une galerie de portraits d'illustres inconnus se retrouvant acteurs d'une histoire hors norme. Particulièrement révélateur est l'engouement des journalistes pour un personnage anonyme, Frédéric Durand, ronéotypiste d'un obscur journal de province dans le « civil » et entomologiste amateur passionné qui avait pris une année sabbatique pour travailler et participer au projet. L'explication fournie alors par les spécialistes de communication était que le public adorait l'idée qu'un « monsieur toutle-monde » soit ainsi mis en avant, le corollaire étant qu'ils pourraient eux-mêmes être amenés - qui sait - à vivre des aventures similaires. Au besoin de héros des années 1980/1990 répond un plébiscite d'anti-héros des années 2000.

Pour un ensemble de raisons qui n'ont pas toutes à voir avec une nouvelle éthique ou avec un intérêt renouvelé des interlocuteurs pour la chose scientifique ou pour la nature, le contenu/les objectifs/ le « dans quel but » ou le « quel impact» sont passés au premier plan. Et il devient évidemment difficile (le temps d'attention et la capacité d'écoute des partenaires financiers et médiatiques n'ayant, eux, pas évolué) de vendre facilement des projets aux contenus flous, peu cohérents et impossibles à résumer dans un «pitch ». Les projets d'opérations qui peuvent mettre en avant 
leurs objectifs scientifiques (généralement ceux qui ont été pensés " science " avant tout autre chose) sont désormais globalement plus à même de capter un auditoire. Il est plus facile de médiatiser - rendre attractive - une opération scientifique, surtout si elle contient en germe une part de rêve et d'aventures exotiques, que de mettre de la « science » dans une opération médiatique, construite et basée essentiellement sur les ressorts de «l'évènementiel ».

\section{De la source aux médias : la fabrique de l'in- formation}

\section{Une communication qui se professionnalise au} Muséum National d'Histoire Naturelle

La comparaison entre les expéditions Panglao (2004), Santo et Mozambique/Madagascar (2010) montre que, en l'espace de cinq ans, le Muséum a considérablement professionnalisé ses services et ses stratégies de communication qui, au début des années 2000, n'étaient en rien comparables avec ce qu'ils sont aujourd'hui. L'année 2003 est marquée par la mise en place d'une grande réforme du Muséum qui, en 2004, se dote notamment « d'outils de communication nouveaux (documents institutionnels) et d'un chargé de mission dédié à la communication scientifique, ce qui jette les bases d'une agence de presse intégrée sur la biodiversité» (MNHN 2004 : 95).

«Parmi les médiateurs « naturels » de la science, outre les chercheurs et les journalistes, il faut souligner l'importance d'une autre catégorie d'acteurs, moins connus du public, mais dont le rôle, situé en amont, est fondamental. Qu'on les appelle chargés de communication, attachés scientifiques, attachés de presse ou spécialistes de l'audiovisuel, ces "médiateurs institutionnels" naviguent à l'interface de deux univers : l'institution, à laquelle ils appartiennent et le monde médiatique » (Tristani-Potteaux 2001 : 7).

L'apparition et la professionnalisation de ces nouveaux métiers de la communication est un phénomène de grande ampleur qui concerne tous les secteurs de la société, et notamment les domaines économiques et politiques (Gorius, Moreau 2011). Dans son ouvrage sur les journalistes scientifiques, Françoise Tristani-Potteaux (1997 : 84) souligne que les budgets et les effectifs des services de communication des organismes de recherche ont décuplé des années 1970 à la fin des années 1990 :

"Comme les entreprises, les organismes de recherche ont appris à maîtriser les outils de la communication, à élaborer une information calibrée, prédigérée, à sortir des communiqués prêts à l'emploi, à nourrir la presse de publications régulières, à fournir les articles clés en main [...]. Ils ont appris à cibler les publics, à sensibiliser les médias adéquats, à maîtriser l'évènement, quitte à le créer, exerçant ainsi une véritable fonction d'agenda, rythmant l'actualité de conférences de presse, célébrations et autres colloques » (Tristani-Potteaux 1997 : 84).

Cette fonction d'agenda est particulièrement visible lorsque l'on compare les expéditions naturalistes étudiées ici. C'est généralement la rédaction d'un communiqué de presse, suivi d'une conférence de presse et de la diffusion d'un dossier de presse qui alertent et informent les médias qui, ensuite, vont décider, ou non, 
de relayer l'information et de couvrir l'événement. Or, l'expédition Panglao n'a bénéficié d'aucune stratégie de communication en amont - ni communiqué ni conférence de presse. En conséquence, la couverture médiatique a été très succincte : trois articles dans la presse spécialisée en plongée sous-marine (Subaqua et Apnéa), un article dans Terre Sauvage, une dépêche AFP, deux articles dans la presse quotidienne régionale et trois articles sur internet.

Avec l'expédition Santo, les choses changent. Elle bénéficie d'une couverture médiatique sans précédent pour ce genre d'opérations : 147 « retombées presse $»^{5}$ de mai 2006 à décembre 2007. Le service de communication du Muséum a tenu une revue de presse de l'expédition, en copiant et archivant les retombées presse. Ce succès médiatique est lié à l'existence d'une stratégie de communication élaborée en amont de l'expédition. Un dossier de presse a été diffusé et des conférences de presse organisées pour le lancement de l'expédition et au retour des participants. Un contrat a été passé avec une agence spécialisée : l'agence Atom ${ }^{6}$.

L'expédition Santo marque un tournant et une rupture en matière de médiatisation des grandes expéditions naturalistes. Au cours d'un entretien effectué en mai 2010 à Madagascar, le photographe officiel de l'expédition Mozambique/ Madagascar, Xavier Desmier qui a participé à l'élaboration du plan médias raconte :

«Pour Santo, j'avais essayé de contacter Philippe Bouchet en 2004 qui rentrait des Philippines (Panglao), ils avaient fait quelque chose pour Terre Sauvage. Mais à l'époque, Philippe n'était pas encore trop sur la communication. Il sentait qu'il y avait une nécessité mais ce n'était absolument pas sa priorité. Je pense que ça ne l'est toujours pas vraiment mais il est plus conscient de l'obligation de faire de la communication pour pouvoir monter de grosses expéditions comme ici à Madagascar. Donc, je n'avais pas vraiment eu de réponse. Et j'avais été voir les responsables de la communication du Muséum. Mais c'était un groupe qui redémarrait tout juste et qui ne s'était pas trop mis en place » (entretien avec Xavier Desmier, Madagascar, mai 2010).

Comme pour les deux expéditions ultérieures (Mozambique 2009 et Madagascar 2010), la campagne médiatique de l'expédition Santo se structure autour du plan média élaboré par les organisateurs : la majorité des articles et des émissions radios et télés se concentrent dans un délai de dix à quinze jours après les conférences de presse de présentation des expéditions puis de retour de ces missions.

Trois ans plus tard, la stratégie de communication, le plan médias et la revue de presse de l'expédition Mozambique/Madagascar se sont encore davantage sophistiqués et professionnalisés. Le Muséum a fait appel à une agence spécialisée en matière de relations presse, The Desk, qui a organisé les conférences de presse, élaboré les dossiers de presse, et fourni un document numérique très complet de 343 pages, organisant, comptabilisant et comportant toutes les retombées presse (167) de ces événements entre octobre 2009 et novembre 2010. Les retombées presse sont listées (noms des revues, radios, télés, sites internet), classées et hiérarchisées dans différentes rubriques (Retombées Audio-Visuelles ; 
Agences de Presse ; Presse Ecrite Nationale ; Presse Ecrite Régionale ; Presse en Ligne), avec les dates de parution et d'émission. Pour les émissions radio et télé, l'heure et la durée sont précisées; pour les articles, la surface de l'article en centimètres carrés est mentionnée. Pour les sites internet, une brève évaluation du site est faite avec le public ciblé et le dynamisme du site mesuré en nombre moyen de pages nouvelles sur une semaine.

\section{L'omniprésence des sources d'information autorisées et officielles}

Élaborés par des agences de communication professionnelles, à la demande des organisateurs des expéditions, les dossiers de presse sont destinés aux médias. Généralement, le travail de ces derniers consiste à faire une sélection des passages qu'ils vont utiliser (en l'état) pour la rédaction de leurs articles. Ces pratiques sont largement répandues dans la sphère journalistique, comme le souligne à juste titre Emmanuel Henry :

« Contrairement à un imaginaire largement répandu, un journaliste effectue surtout un travail de réception et de tri d'informations provenant soit d'autres médias d'information ou d'agences de presse, soit de sources d'information de plus en plus professionnalisées qui lui envoient régulièrement 'dossiers de presse'ou 'communiqués' » (Henry 2003 : 253).

Les dossiers de presse constituent donc des documents essentiels qui orientent et déterminent le contenu de la médiatisation de ces événements. Les sources d'information donnent donc non seulement le tempo de la couverture médiatique mais aussi le ton et le contenu. Hormis certains médias qui diversifient parfois leurs sources - et notamment ceux que l'on appelle les « médias acquis » avec lesquels sont passés, en amont de l'expédition des contrats (parfois d'exclusivité) - les médias se contentent soit de reprendre les documents officiels (communiqués et dossiers de presse), soit d'interviewer les personnes considérées comme autorisées et légitimes, à savoir les organisateurs eux-mêmes, le directeur général et le président du Muséum.

Pourtant, la majorité des participants de ces expéditions travaillent à Paris, donc à proximité des journalistes. Ce n'est donc ni l'éloignement ni le coût qui explique l'absence d'interviews auprès d'une plus grande diversité d'interlocuteurs, comme cela était a contrario le cas des journalistes dans l'enquête réalisée par Emmanuel Henry sur la médiatisation de l'amiante (Henry 2003 : 250). L'utilisation de ce qui est considérée comme une source d'information autorisée et donc légitime est une pratique journalistique habituelle, comme le rappelle Philip Schlesinger (1992: 84) :

«La découverte essentielle d'une grande partie de la sociologie du journalisme est que la pratique journalistique favorise généralement les intérêts des sources faisant autorité, notamment celles qui se situent à l'intérieur de l'appareil gouvernemental et étatique. »

Seuls les rares journalistes qui participent aux expéditions diversifient davantage leurs sources d'information et offrent des regards plus variés sur les expéditions. Leur présence a été négociée en amont, et ils font partie des « médias acquis ». 


\section{" Acquérir " des médias}

Il y a en effet deux types de médias : les « médias acquis " et les «médias conquis ". Le succès d'une opération peut être en partie anticipé. Une partie du succès médiatique est acquis $a$ priori : on le mesure aux «préventes » dans différents médias.

Pour l'expédition Mozambique/Madagascar, comme pour l'expédition Santo, un plan média a été élaboré en amont. Xavier Desmier, qui s'est occupé de planifier et d'organiser la couverture presse raconte qu'ils ont contacté en premier le magazine National Geographic parce que cela correspondait bien à l'expédition et qu'en plus, il les connaissait bien pour avoir beaucoup travaillé avec eux. Mais pour des raisons financières, National Geographic n'a pas pu couvrir l'évènement. En effet, le budget presse n'est pas pris sur le budget de l'expédition. C'est aux journaux de financer eux-mêmes leurs reportages. Or dans le contexte actuel de crise des médias, nombreux sont ceux qui n'en ont pas les moyens. Xavier Desmier se tourne alors vers le Figaro Magazine dont il connaît très bien le rédacteur photo :

«J'avais vu le rédacteur photo du Figaro avec qui je travaille régulièrement qui, en plus, est un ami. L'avantage du Figaro pour l'expédition c'est un magazine qui est diffusé à $300000 \mathrm{ou}$ 350000 exemplaires mais surtout qui est lu par 2 millions de lecteurs. C'est quand même un magazine phare et c'est un hebdo en plus, et qui, notamment par rapport à des partenaires financiers, est intéressant parce qu'il est classé $\grave{a}$ «droite ». Sans rentrer dans les histoires politiques, c'est quand même un magazine qui tient la route, en photos et en textes aussi. Le Figaro nous proposait 12 pages minimum, 20 maxi et la couverture » (entretien avec Xavier Desmier, Madagascar mai 2010).

De plus en plus, la couverture médiatique est organisée en amont de l'opération, des accords sont passés avec différents représentants de la presse écrite (quotidienne, mensuelle, etc.) et audiovisuelle (production de documentaires, rendez-vous pour des passages radio). Il est donc tentant, dès les prémisses d'un projet, de le modifier en conséquence, voire de le monter de toutes pièces en fonction des besoins / attentes médiatiques. Pour autant, ce sont les médias « conquis » qui sanctionneront a posteriori - en fonction de leurs attentes et de leur vision de l'opération - la force de son succès. Toutes choses égales par ailleurs (en l'occurrence une capacité équivalente à communiquer), c'est bien l'adéquation avec les attentes du moment qui dictera le succès journalistique, et donc public, de l'opération.

\section{Conclusion}

Lorsqu'on analyse le contenu des articles de presse sur les expéditions, on constate que les journalistes choisissent de sensibiliser le public en se focalisant sur trois principales dimensions : les menaces qui pèsent sur la biodiversité ; la découverte de nouvelles espèces ; le voyage contemporain et historique.

Dans son article intitulé : « Biodiversité : voici les héritiers de Buffon », la journaliste du Figaro Magazine, qui a suivi, sur le terrain, les expéditions Mozambique/Madagascar écrit : 
"Imaginé par le Muséum national d'histoire naturelle et l'ONG Pro-Natura, le projet «La planète revisitée » remet au goût du jour les grandes expéditions naturalistes. Partis à la découverte des forêts côtières du Mozambique et des fonds sous-marins du Grand Sud de Madagascar, des dizaines de scientifiques travaillent à un vaste inventaire de la biodiversité de notre planète » (Clarens 2010 : 52).

Dans son éditorial daté de mars 2007, JeanJacques Fresko, rédacteur en chef de Terre Sauvage écrit :

«Par son ampleur - 25 pays représentés -, par son enjeu - l'inventaire de la biodiversité de l'île avant que les effets du changement climatique ne la ravagent - par ses premiers résultats, Santo 2006 est à la hauteur des plus grandes expéditions historiques » (Fresko $2007: 5$ ).

La liste est longue d'extraits d'articles qui reprennent d'une manière ou d'une autre ces thématiques. Tous les ingrédients sont ainsi réunis pour donner de la grandeur, historique, scientifique et politique à ces expéditions. De par leurs enjeux (inventorier la biodiversité et la protéger avant qu'elle ne disparaisse), leurs résultats (découvrir de nouvelles espèces vivantes et les mettre en collections dans les grands muséums de la planète) et les modèles dont elles héritent (les plus grands explorateurs de tous les temps), ces missions naturalistes, sous la plume des journalistes, parviennent à emporter les lecteurs et téléspectateurs dans des aventures uniques, menées pourtant par des « scientifiques ordinaires ».

Quel est l'impact de cette médiatisation sur l'émergence d'une opinion publique en matière d'environnement en général et d'érosion de la biodiversité en particulier ? Même si nous n'avons pas fait "d'enquêtes d'opinion » ni d'enquêtes qualitatives auprès des lecteurs et téléspectateurs des médias mobilisés ici, les choix narratifs privilégiés par les journalistes ont forcément un effet sur la formation des « publics de la biodiversité ».

Comme le montrent certains travaux (Comby 2008 et 2009), certaines thématiques environnementales, comme les questions climatiques, ont réussi à trouver un écho important dans la société civile et auprès des responsables politiques. À ce jour, on serait tenté de dire qu'il n'en va pas de même pour l'érosion de la biodiversité et de la disparition annoncée comme inéluctable de milliers d'espèces, végétales et animales. Pourtant, le fait que les organisateurs de « La Planète Revisitée " parviennent à trouver des mécènes pour financer leurs opérations, et des grands médias nationaux pour couvrir l'événement semble révéler l'émergence d'une opinion publique en matière de biodiversité, et ce, même lorsqu'il est question, comme ici, d'invertébrés terrestres et marins, c'est-à-dire d'espèces qui ne sont pas emblématiques et pour lesquelles il est difficile de provoquer de l'empathie.

Il faut dire que les histoires racontées par les journalistes - qui reprennent les discours des communicants - mêlant aventures, découvertes, voyages et menaces sont efficaces : comme la journaliste de Terre Sauvage qui, en 2006, comparait son arrivée à Santo avec celle, 400 ans plus tôt, de Pedro Fernandez de Quiros, navigateur portugais qui découvrit, pour les Européens, 
l'île de Santo (Lescroart 2007 : 16). Se rêvant explorateurs des temps modernes, ils emportent avec eux le lecteur et le téléspectateur dans ces Grandes Découvertes.

\section{$R \cdot E ́ E \cdot F \cdot E \cdot R \cdot E \cdot N \cdot C \cdot E \cdot S$}

Katia CLARENS, « Voici les héritiers de Buffon » in Le Figaro Magazine, 21 mai, 2010, pp. 52-62.

Jean-Baptiste COMBY, « Quand l'environnement devient « médiatique ». Conditions et effets de l'institutionnalisation d'une spécialité journalistique », in Réseaux, $n^{\circ}$ 157-158, 2009, pp. 157-190.

Jean-Baptiste COMBY, Créer un climat favorable. Les enjeux liés au changement climatique : valorisation publique, médiatisation et appropriation au quotidien, Paris II, Thèse de doctorat en sciences de l'information et de la communication, 2008, p. 456.

Jean-Jacques FRESKO, «Quelles nouvelles, monsieur Le Guyader ? ", in Terre Sauvage, mars 2007, p. 5.

Aurore GORIUS, Michaël MOREAU, Les gourous de la com'. Trente ans de manipulations politiques et économiques, Paris, La Découverte, 2011, p. 311.

Emmanuel HENRY, « Du silence au scandale. Des difficultés des médias d'information à se saisir de la question de l'amiante », in Réseaux, $\mathrm{n}^{\circ} 122,2003$, pp. 237-272.

Marie LESCROART, « Santo 2006 : grand inventaire dans l'île aux trésors », in Terre Sauvage, mars 2007, pp. 12-35.

MUSÉUM NATIONAL D'HISTOIRE NATURELLE, Rapport d'activités, Paris, 2004, p. 144. Philippe SCHLESINGER, « Repenser la sociologie du journalisme. Les stratégies de la source d'information et les limites du média-centrisme », in Réseaux, vol 10, n 51, 1992, pp. 75-98.

Françoise TRISTANI-POTTEAUX, « Du labo- 
ratoire au citoyen, les trois étapes de la communication scientifique ", in CNRS Info, $\mathrm{n}^{\circ} 394$, 2001, pp. 7-8.

Françoise TRISTANI-POTTEAUX, Les journalistes scientifiques, médiateurs des savoirs, Paris, Economica, 1997, p. 110.
$\mathrm{N} \cdot \mathrm{O} \cdot \mathrm{T} \cdot \mathrm{E} \cdot \mathrm{S}$

1. À la suite de l'expédition Santo, les organisateurs élaborent un programme de dix ans d'expéditions naturalistes, dans les hotspots de la biodiversité, centré sur les taxons négligés (invertébrés terrestres et marins) appelé «La Planète Revisitée ». Ce programme est étudié par des chercheurs en sciences sociales dans le cadre d'un projet de recherche intitulé « Expébiodiv. Étude pluridisciplinaire des grandes expéditions naturalistes contemporaines » financé par l'Agence Nationale de la Recherche et coordonné par Elsa Faugère.

2. Les principaux mécènes des expéditions Panglao, Santo et Mozambique/Madagascar sont les fondations Total, Stavros Niarchos et Prince Albert II de Monaco. 3. Nous remercions ici Philippe Maestrati et Estelle Merceron (DICAP Muséum) de nous avoir transmis les revues de presse de ces expéditions.

4. Cette proposition de chronologie se fonde notamment sur les connaissances et l'expérience professionnelles d'Olivier Pascal qui, depuis la fin des années 1980, a co-organisé de nombreuses expéditions naturalistes, d'abord dans le cadre du Radeau des Cimes puis, dans les années 2000 dans le cadre de La Planète Revisitée.

5. On appelle retombée presse tout événement médiatique, de presse écrite ou audiovisuelle.

6. Maintenant « Docside production».

Mots-clefs : expédition naturaliste, journalisme environnemental, médias, professionnels de la communication, chaîne de l'information.

Key Words : naturalistic expedition, environmental journalism, media, communication, information 


\title{
$R \cdot E ́ \cdot S \cdot U \cdot M \cdot E ́$
}

En 2006, une grande expédition naturaliste se déroule au Vanuatu, sur l'île de Santo. Organisée par le Muséum National d'Histoire Naturelle de Paris, ProNatura International et l'Institut de Recherches pour le Développement, financée par des mécènes privés, elle va connaître un succès médiatique sans précédent pour ce genre d'opérations. Elle inaugure un nouveau type d'expéditions scientifiques caractérisées par des stratégies de communication efficace, condition nécessaire pour bénéficier d'une bonne couverture médiatique recherchée par les mécènes. Dans ce contexte de professionnalisation du journalisme environnemental, nous nous intéressons ici à la chaîne de production de l'information depuis les sources jusqu'à leur traitement médiatique. Nous verrons ainsi que les sources elles-mêmes - les institutions et acteurs organisateurs des expéditions- diffusent les ingrédients nécessaires pour donner de la grandeur, historique, scientifique et politique à ces expéditions.

\begin{abstract}
In 2006, the National Museum of Natural History of Paris, Pro-Natura International and the Research Institute for Development, organized a broad naturalistic expedition in Santo's island, in Vanuatu. Supported by private foundations, this expedition had an unprecedented media success. It marks the start of a new kind of scientific operations with an efficient strategy of communication, which leads to a good media coverage researched by the sponsors. In this context of the professionalization of environmental journalism, this paper is focused on the production of information, from sources to medias. It shows that ingredients in the sources themselves give historical, scientific, and political greatness to those expeditions.
\end{abstract}


